\title{
Nutritional Assessment in Completely Edentulous Geriatric Patients
}

\author{
Krishna Prasad D ${ }^{1}$, Farah Demeri ${ }^{2}$, Chethan Hegde ${ }^{3}$, Anupama Prasad $D^{4}$
}

\begin{abstract}
Aims and objectives: The relationship between nutrition and oral health in geriatric patients is controversial and complex. It has been suggested that poor oral health leads to a decreased ability to chew having profound influence on diet, food selection, and therefore affect the nutritional status of an individual. This study aimed to evaluate the effect of edentulousness on the nutritional health of geriatric patients.

Materials and methods: A total of 200 completely edentulous geriatric patients were selected for the study. The nutritional status was assessed using the mini-nutritional assessment (MNA) form before the complete denture treatment. The individual scores obtained for each question based on which the total score was determined. Correct responses of each question were calculated in percentage.

Results: The results showed statistically insignificant differences in the total MNA scores between males and females in the study. The edentulous patients without complete dentures had significantly lower MNA scores and $88.5 \%$ of the study group were at risk of malnourishment.

Conclusion: Prosthetic rehabilitation of the edentulous subjects, along with the dietary counseling can improve the nutritional status of geriatric complete denture patients as it will enhance the chewing ability and function.

Clinical significance: Early assessment of nutritional status in completely edentulous patients can permit an early nutritional intervention when necessary so as to prevent malnutrition.

Keywords: Counseling, Edentulous, Geriatrics, Mini-nutritional assessment, Nutrition.

World Journal of Dentistry (2020): 10.5005/jp-journals-10015-1742
\end{abstract}

\section{INTRODUCTION}

Diet is a necessary element to lead a healthy life, since it plays a role in the etiology and aids in the management and prevention of chronic conditions that include obesity, cardiovascular disease, diabetes, and cancer. ${ }^{1,2}$ The oral cavity which primarily aids in mastication is an entryway for nutrient consumption. ${ }^{3}$ Tooth loss decreases chewing ability and masticatory function, thus limiting food choices and variety in the person's diet. ${ }^{4,5}$

Elderly patients suffer from various diseases that tend to impair their nutritional status. ${ }^{6}$ In addition to chronic diseases, they also suffer from loss of teeth. Russia and Mexico have edentulous population of about 16.3-21.7\%, Ghana, South Africa and, China is about $3.0-9.0 \%$, whereas, in India, it is $11.7 \%$. Successful prosthodontic treatment in the geriatric edentulous population is necessary for the general health of oral hard and soft tissues. ${ }^{7}$

The elderly population suffers from malnutrition, and it is a health problem having multifactorial parameters. These include social, economic, and physiological parameters. These parameters are referred to as the $9 \mathrm{~d}$ 's, namely dysgeusia, diarrhea, poor dentition, disease, dysphagia, depression, dysfunction, drugs, and dementia. ${ }^{8}$ Early diagnosis and treatment of malnutrition is the key to stop this vicious cycle. ${ }^{9}$

In order to recognize malnutrition at an early stage, various nutritional screening tools have been proposed. These screening tools are based mainly on clinical, nutritional, and biochemical indexes. The mini-nutritional assessment (MNA) form is based on anthropometric, general, dietary, and self-assessment by the patients. ${ }^{10}$

The aims of the present survey were to assess the nutritional status in geriatric patients who are completely edentulous and to evaluate their risk of malnutrition. This will enable us in the early nutritional intervention when needed using the MNA.
${ }^{1-4}$ Department of Prosthodontics and Crown and Bridge, AB Shetty Memorial Institute of Dental Sciences, NITTE (Deemed to be University), Mangaluru, Karnataka, India

Corresponding Author: Anupama Prasad D, Department of Prosthodontics and Crown and Bridge, AB Shetty Memorial Institute of Dental Sciences, NITTE (Deemed to be University), Mangaluru, Karnataka, India, Phone: +91 9448504764, e-mail: dranupamaprasad@ nitte.edu.in

How to cite this article: Prasad DK, Demeri F, Hegde C, et al. Nutritional Assessment in Completely Edentulous Geriatric Patients. World J Dent 2020;11(4):284-286.

Source of support: Nil

Conflict of interest: None

\section{Materials and Methods}

Two hundred completely edentulous subjects visiting the outpatient department of our institute were selected for this survey. The patients gave a written consent prior to the study. The MNA questionnaire was filled out by the examiner for each patient. The assessment included anthropometric, general, dietary, and self-assessment. The patients were asked questions in their native language for ease of understanding. Only completely edentulous subjects were considered in the survey. The minimum period of edentulousness for each subject included was $>1$ year. Patients less than the age of 65 years were not considered in the study. The study excluded patients who wore complete dentures for more than a year. The results of the study were compiled and statistical analysis was conducted. The individual responses were subjected to percentage statistics, and student $t$-test was applied. 


\section{Results}

The total score was calculated for each question in the questionnaire. Eleven percent patients showed a body mass index (BMI) below 19, which is considered to be underweight. $34.5 \%$ had a BMI of $19-21$, and $53 \%$ had a BMI of $21-23$, which is considered to be in the normal range. $1.5 \%$ of patients were considered obese (Table 1). Table 2 measures the mid-arm circumference, of which $48 \%$ of patients showed a mid-arm circumference of $21-22 \mathrm{~cm}, 40.5 \%$ more than $22 \mathrm{~cm}$, and $11.5 \%$ less than $21 \mathrm{~cm}$. Measured calf circumference of more than $31 \mathrm{~cm}$ was seen in $62.5 \%$ patients and less than $31 \mathrm{~cm}$ in $37.5 \%$ patients (Table 3). Table 4 shows the total assessment after the final calculation of points by the examiner. In our study, the wellnourished population constituted of only $1 \%$ sample. $88.5 \%$ of the study population were at increased risk of malnutrition, and $10.5 \%$ were malnourished. Ninety-six males and 104 females were a part of the study. The mean malnutrition score for males and females was 19.573 and 19.567 , respectively, indicating that both groups were at risk of malnutrition (Table 5). However, these results were statistically not significant, with a $p$ value of 0.98 . Table 6 shows $1 \%$ of males and females were malnourished. $90.6 \%$ of males and $86.5 \%$ of females are at risk of malnutrition, whereas $8.3 \%$ of males and $12.5 \%$ of females are malnourished. These results are statistically not significant, with a $p$ value of 0.63 .

\section{Discussion}

The validation and development of the MNA was a joint research program between the Department of Internal Medicine and Clinical

Table 1: Measurement of body mass index

\begin{tabular}{lcc}
\hline & Frequency & Percent \\
\hline$<19$ & 22 & 11.0 \\
$19-21$ & 69 & 34.5 \\
$21-23$ & 106 & 53.0 \\
$\geq 23$ & 3 & 1.5 \\
Total & 200 & 100.0 \\
\hline
\end{tabular}

Table 2: Measurement of mid-arm circumference in centimeter

\begin{tabular}{lcc}
\hline & Frequency & Percent \\
\hline$<21 \mathrm{~cm}$ & 23 & 11.5 \\
$21-22 \mathrm{~cm}$ & 96 & 48.0 \\
$>22 \mathrm{~cm}$ & 81 & 40.5 \\
Total & 200 & 100.0 \\
\hline
\end{tabular}

Table 3: Measurement of calf circumference in centimeter

\begin{tabular}{lcc}
\hline & Frequency & Percent \\
\hline$<31 \mathrm{~cm}$ & 75 & 37.5 \\
$\geq 31 \mathrm{~cm}$ & 125 & 62.5 \\
Total & 200 & 100.0 \\
\hline
\end{tabular}

Table 4: Total malnutrition assessment

\begin{tabular}{lcc}
\hline & Frequency & Percent \\
\hline Well nourished & 2 & 1.0 \\
At risk of malnutrition & 177 & 88.5 \\
Malnourished & 21 & 10.5 \\
Total & 200 & 100.0 \\
\hline
\end{tabular}

Gerontology, Toulouse University Hospital, France, the Clinical Nutrition Program, University of New Mexico, USA, and the Nestlé Research Center, Lausanne, Switzerland. ${ }^{11}$ This test contains a series of questions and measurements that consist of measurements of weight, weight loss, and height (anthropometric measurements), six questions on global assessment which include mobility, medication and lifestyle, subjective perception of health and nutrition, and eight questions related to the diet of the patient. ${ }^{9}$ Many studies have identified MNA as a tool for early identification of malnutrition, and thereby helping us in the early intervention of the disease process. ${ }^{12}$

Nutritional status of a patient can be designated as good when the MNA score is $\geq 24$. Information should be provided to these patients about how to remain healthy and maintain nourishment. If the patients experience weight loss they should reported it to their concerned physician. Geriatric patients should have a flexible diet and not restrict food consumptions to a cholesterol-restricted diet. A MNA score of 17 or below is indicative of protein-calorie deficit. $^{2-4}$ These individuals experience weight loss and present with decreased serum albumin. For these patients, a thorough nutritional assessment is indicated. This measurement helps us to identify and study the disease cause and start treatment to increase overall health and nourishment. ${ }^{13}$ Mini-nutritional assessment scores between 17 and 23.5 are defined as the people who are at risk of malnutrition. Serum albumin levels may be normal in these individuals and they may not experience weight loss. However, these patients show a reduction in caloric intake. This calorie deficit can be corrected by an adequate nutrition. ${ }^{2-4}$

Poor oral status, especially edentulism, affects the nutritional status and overall health. Difficulty in chewing impairs the functional capacity and thereby overall health. With an increase in age, people tend to increase the number of chewing strokes to form a bolus for swallowing. Age is not the only determinant of masticatory ability. The extent to which a patient loses his/her teeth can affect the chewing performance and the selection of food. ${ }^{14}$

Prosthetic rehabilitation plays a key role in affecting nutritional status of edentulous individuals. Edentulousness causes a decline in masticatory ability, which is compensated by many patients by choosing cooked foods or processed foods rather than fresh

Table 5: Group statistics

\begin{tabular}{llrlll}
\hline & Gender & $N$ & Mean & Std. deviation & $t$ \\
\hline Total & Male & 96 & 19.573 & 1.906 & 0.021 \\
& Female & 104 & 19.567 & 1.799 & $p=0.983 \mathrm{~ns}$ \\
\hline$p=0.983$ (not significant) & & &
\end{tabular}

Table 6: Total assessment and gender

\begin{tabular}{|c|c|c|c|c|}
\hline & & \multicolumn{2}{|c|}{ Gender } & \multirow[b]{2}{*}{ Total } \\
\hline & & Male $e^{a}$ & Female & \\
\hline \multirow[t]{2}{*}{ Well nourished } & Count & 1 & 1 & 2 \\
\hline & $\%$ & 1.0 & 1.0 & 1.0 \\
\hline \multirow[t]{2}{*}{ At risk of malnutrition } & Count & 87 & 90 & 177 \\
\hline & $\%$ & 90.6 & 86.5 & 88.5 \\
\hline \multirow[t]{2}{*}{ Malnourished } & Count & 8 & 13 & 21 \\
\hline & $\%$ & 8.3 & 12.5 & 10.5 \\
\hline \multirow[t]{2}{*}{ Total } & Count & 96 & 104 & 200 \\
\hline & $\%$ & 100.0 & 100.0 & 100.0 \\
\hline
\end{tabular}

${ }^{\mathrm{a}} X^{2}=0.923, p=0.63 \mathrm{~ns}$ 
foods. These individuals have a diet that is low in non-starch polysaccharides and also has difficulty in eating some forms of bread, vegetables, and fruits. Due to an imbalance in food intake and/or reduced nutrient bioavailability, gastrointestinal disturbance can be the end result.

Studies have shown that prosthodontically if we rehabilitate these edentulous patients with a complete denture prosthesis, it can improve the diet quality and food choices in these geriatric patients. However, few studies point out to the fact that some rehabilitation does not necessarily change the diet pattern of geriatric individuals. $5,15,16$

Our study pointed out that majority of the patients were at risk of malnutrition $(88.5 \%), 10.5 \%$ were found to be malnourished, and $1 \%$ were well-nourished. Thus, oral health status and nutrition are said to be related. Before treatment with complete dentures, edentulous patients show lower MNA scores when compared to those who have been rehabilitated. Studies indicate that rehabilitation with complete denture counteracts the effect of missing teeth. Individuals who have a higher MNA score have increased eating pleasure and reduced eating difficulties. ${ }^{17}$

Nutritional deficiency has a multifactorial etiology. Mental physical and general health, psychological habits, and socioeconomic status are the factors affecting the diet habits of an individual. The fourth factor associated with diet selection is oral health status, which is usually affected in geriatric individuals. ${ }^{17,18}$ By including these factors, this study was carried out by utilizing the MNA.

\section{Conclusion}

Within the limitations of this study, it can be concluded that:

- Poor oral health status affects the ability in eating food and also decreases eating pleasure.

- There is a higher risk of undernutrition associated with edentulism.

- Dietary counseling and rehabilitation of completely edentulous patients can be performed in order to improve their nutritional intake and function.

\section{Clinical Significance}

Early assessment of nutritional status in completely edentulous patients can permit an early nutritional intervention when necessary so as to prevent malnutrition.

\section{ACKnOWLedgment}

Our acknowledgment extends to AB Shetty Memorial Institute of Dental Sciences, NITTE (Deemed to be University), Deralakatte, Mangaluru, Karnataka, India-575018.

\section{References}

1. Who J, Consultation FE. Diet, nutrition and the prevention of chronic diseases. World Health Organ Tech Rep Ser 2003;916(i-viii).

2. Willett WC. Diet and health: what should we eat? Science 1994;264(5158):532-537. DOI: 10.1126/science.8160011.

3. Lee IC, Yang YH, Ho PS, et al. Chewing ability, nutritional status and quality of life. J Oral Rehabil 2014;41(2):79-86. DOI: 10.1111/ joor.12115.

4. Sheiham A, Steele JG, Marcenes W, et al. The relationship among dental status, nutrient intake, and nutritional status in older people. J Dent Res 2001;80(2):408-413. DOI: 10.1177/00220345010800020201.

5. Nowjack-Raymer RE, Sheiham A. Association of edentulism and diet and nutrition in US adults. J Dent Res 2003;82(2):123-126. DOI: $10.1177 / 154405910308200209$.

6. Edington J, Boorman J, Durrant ER, et al. Prevalence of malnutrition on admission to four hospitals in England. The malnutrition prevalence group. Clin Nutr 2000;19(3):191-195. DOI: 10.1054/clnu. 1999.0121.

7. Peltzer K, Hewlett $\mathrm{S}$, Yawson A, et al. Prevalence of loss of all teeth (edentulism) and associated factors in older adults in China, Ghana, India, Mexico, Russia and South Africa. Int J Environ Res Public Health 2014;11(11):11308-11324. DOI: 10.3390/ijerph111111308.

8. Agarwal E, Miller M, Yaxley A, et al. Malnutrition in the elderly: a narrative review. Maturitas 2013;76(4):296-302. DOI: 10.1016/j. maturitas.2013.07.013.

9. Vellas B, Guigoz Y, Garry PJ, et al. The mini nutritional assessment (MNA) and its use in grading the nutritional state of elderly patients. Nutrition 1999;15(2):116-122. DOI: 10.1016/s0899-9007(98) 00171-3.

10. Cereda E, Pedrolli C. The geriatric nutritional risk index. Curr Opin Clin Nutr Metab Care 2009;12(1):1-7. DOI: 10.1097/MCO.0b013e3283186f59.

11. Guigoz $\mathrm{Y}$. The mini nutritional assessment $\left(\mathrm{MNA}^{\oplus}\right)$ review of the literature-what does it tell us? J Nutrit Health Aging 2006;10(6):466.

12. Reber E, Gomes F, Vasiloglou MF, et al. Nutritional risk screening and assessment. J Clin Med 2019;8(7):1065. DOI: 10.3390/ jcm8071065.

13. Bharadwaj S, Ginoya S, Tandon P, et al. Malnutrition: laboratory markers vs nutritional assessment. Gastroenterol Rep (Oxf) 2016;4(4):272-280. DOI: 10.1093/gastro/gow013.

14. Cichero JA. Age-related changes to eating and swallowing impact frailty: aspiration, choking risk, modified food texture and autonomy of choice. Geriatrics 2018;3(4):69. DOI: 10.3390/geriatrics3040069.

15. Krall E, Hayes C, Garcia R. How dentition status and masticatory function affect nutrient intake. J Am Dent Assoc 1998;129(9):12611269. DOI: 10.14219/jada.archive.1998.0423.

16. Budtz-Jørgensen E, Chung JP, Rapin $\mathrm{CH}$. Nutrition and oral health. Best Pract Res Clin Gastroenterol 2001;15(6):885-896. DOI: 10.1053/ bega.2001.0247.

17. Guigoz $Y$, Lauque $S$, Vellas BJ. Identifying the elderly at risk for malnutrition. The mini nutritional assessment. Clin Geriatr Med 2002;18(4):737-757. DOI: 10.1016/s0749-0690(02)00059-9.

18. Cho HS, Lee HR, Yoo GJ, et al. Factors related to nutritional status of elderly in a Korean rural community. J Korean Geriat Soc 1997;1(2): 120-139. 